\title{
Notes on Greub-Rheinboldt inequalities
}

\author{
Di Zhao ${ }^{1}$, Hongyi Li ${ }^{*}$ and Zhiguo Gong ${ }^{2}$
}

"Correspondence: zdhyl2010@126.com

'LMIB, School of Mathematics and System Science, Beihang University, Beijing, China

Full list of author information is available at the end of the article

\begin{abstract}
In this paper, we focus on matrix Greub-Rheinboldt inequalities for commutative positive definite Hermitian matrix pairs. Some improvements, which yield sharpened bounds compared with existing results, are presented.
\end{abstract}

\section{Introduction and preliminaries}

Let $M_{m, n}$ denote the space of $m \times n$ complex matrices and write $M_{n} \equiv M_{n, n}$. The identity matrix in $M_{n}$ is denoted by $I_{n}$. As usual, $A^{*}=(\bar{A})^{T}$ denotes the conjugate transpose of the matrix $A$. A matrix $A \in M_{n}$ is an Hermite matrix if $A^{*}=A$. An Hermitian matrix $A$ is said to be positive semi-definite or nonnegative definite, written as $A \geq 0$, if $x^{*} A x \geq 0, \forall x \in \mathbb{C}^{n}$. $A$ is further called positive definite, symbolized $A>0$, if $x^{*} A x>0$ for all nonzero $x \in \mathbb{C}^{n}$. An equivalent condition for $A \in M_{n}$ to be positive definite is that $A$ is an Hermitian matrix and all eigenvalues of $A$ are positive.

Denote by $\lambda_{1} \leq \lambda_{2} \leq \cdots \leq \lambda_{n}$ the eigenvalues of an Hermitian matrix $A$. The matrix version of the well-known Kantorovich inequality for a positive definite matrix $A$ is stated as follows (see, e.g., [1, 2]):

$$
1 \leq \frac{x^{*} A x x^{*} A^{-1} x}{\left(x^{*} x\right)^{2}} \leq \frac{\left(\lambda_{1}+\lambda_{n}\right)^{2}}{4 \lambda_{1} \lambda_{n}}
$$

for any nonzero vector $x \in \mathbb{C}^{n}$.

An equivalent form of this result is the inequality

$$
0 \leq \frac{x^{*} A x x^{*} A^{-1} x}{\left(x^{*} x\right)^{2}}-1 \leq \frac{\left(\lambda_{1}-\lambda_{n}\right)^{2}}{4 \lambda_{1} \lambda_{n}}
$$

valid for any nonzero vector $x \in \mathbb{C}^{n}$.

This famous inequality plays an important role in statistics (see [3, 4]; for the latest work on applications in statistics, we refer to Seddighin's work [3]) and numerical analysis, for example, studying the rates of convergence and error bounds of solving systems of equations (see in $[5,6])$.

In 2008, Dragomir gave a refinement of the additive version of the operator Kantorovich inequality [7],

$$
0 \leq K(A ; x)-1 \leq \frac{1}{4} \frac{(M-m)^{2}}{m M}-\left[\operatorname{Re}\left(C_{m, M}(A) x, x\right) \operatorname{Re}\left(C_{\frac{1}{m}, \frac{1}{M}}\left(A^{-1}\right) x, x\right)\right]^{1 / 2},
$$


where $A$ is a self-adjoint bounded linear operator on a complex Hilbert space, $0<m<$ $M$, such that $m I \leq A \leq M I$ in the partial operator order, $K(A ; x):=\langle A x, x\rangle\left\langle A^{-1} x, x\right\rangle$, and $C_{\alpha, \beta}(A):=(A-\bar{\alpha} I)(\beta I-A)$.

A further improvement of the matrix version of (1.3) is proposed in [8], where the classical Kantorovich inequality (1.1) is modified to apply not only to positive definite, but also to all invertible Hermitian matrices.

We adopt the following transform for a positive definite Hermitian matrix $A \in M_{n}$ with eigenvalues $0<\lambda_{1} \leq \lambda_{2} \leq \cdots \leq \lambda_{n}$ :

$$
C(A, x)=x^{*}\left(\lambda_{n} I-A\right)\left(A-\lambda_{1} I\right) x,
$$

and

$$
C\left(A^{-1}, x\right)=x^{*}\left(\frac{1}{\lambda_{1}} I-A^{-1}\right)\left(A^{-1}-\frac{1}{\lambda_{n}} I\right) x .
$$

Then the following inequality holds [8]:

$$
0 \leq x^{*} A x \cdot x^{\prime \prime} A^{-1} x-1 \leq \frac{\left(\lambda_{1}-\lambda_{n}\right)^{2}}{4 \lambda_{1} \lambda_{n}}-\sqrt{C(A, x) \cdot C\left(A^{-1}, x\right)} \leq \frac{\left(\lambda_{1}-\lambda_{n}\right)^{2}}{4 \lambda_{1} \lambda_{n}} .
$$

The result above is an improvement of the Kantorovich inequality (1.1).

A generalized form of the Kantorovich inequality presented by Greub and Rheinboldt [1] in 1959 is known as the Greub-Rheinboldt inequality in operator theoretic terms, which is also an important and early example of the so-called complementary inequality referred to in [9],

$$
\langle A x, A x\rangle\langle B x, B x\rangle \leq \frac{\left(M_{1} M_{2}+m_{1} m_{2}\right)^{2}}{4 m_{1} m_{2} M_{1} M_{2}}\langle A x, B x\rangle^{2},
$$

where $A$ and $B$ are commuting positive definite self-adjoint operators on a Hilbert space, with upper and lower bounds $M_{i}$ and $m_{i}, i=1,2$, respectively.

In 1997, Fujii et al. [10] generalized the Greub-Rheinboldt inequality to pairs of invertible operators that may not even commute,

$$
\left\langle A^{2} \sharp B^{2} x, x\right\rangle \leq\left\langle A^{2}, x\right\rangle^{1 / 2}\left\langle B^{2}, x\right\rangle^{1 / 2} \leq \frac{m_{1} m_{2}+M_{2} M_{2}}{2 \sqrt{m_{1} m_{2} M_{1}} M_{2}}\left\langle A^{2} \sharp B^{2} x, x\right\rangle\langle A x, B x\rangle^{2},
$$

where $A, B$ are invertible positive operators satisfying $0<m_{1} \leq A \leq M_{1}$ and $0<m_{2} \leq B \leq$ $M_{2}$, and $A \sharp B=A^{1 / 2}\left(A^{-1 / 2} B A^{-1 / 2}\right)^{1 / 2} A^{1 / 2}$. By using the viewpoint of interaction antieigenvalue, Gustafson [9] sharpened the Greub-Rheinboldt inequality (1.7) to obtain the following result:

$$
\langle A x, A x\rangle\langle B x, B x\rangle \leq \frac{\left(m\left(A B^{-1}\right)+M\left(A B^{-1}\right)\right)^{2}}{4 m\left(A B^{-1}\right) M\left(A B^{-1}\right)}\langle A x, B x\rangle^{2},
$$

where $A$ and $B$ are commuting positive definite self-adjoint operators on a Hilbert space.

Let $A$ and $B$ be two positive definite Hermite matrices and $A B=B A$ with real eigenvalues $\lambda_{1} \leq \lambda_{2} \leq \cdots \leq \lambda_{n}$ and $\mu_{1} \leq \mu_{2} \leq \cdots \leq \mu_{n}$, respectively. Moreover, let $\langle A x, B x\rangle:=$ 
$(A x)^{*} B x=x^{*} A^{*} B x$. Then a matrix version of (1.9) is

$$
\frac{x^{*} A^{2} x \cdot x^{*} B^{2} x}{\left(x^{*} A B x\right)^{2}} \leq \frac{\left(\lambda_{1} \mu_{1}+\lambda_{n} \mu_{n}\right)^{2}}{4 \lambda_{1} \lambda_{n} \mu_{1} \mu_{n}}
$$

for any nonzero vector $x \in \mathbb{C}^{n}$.

In 2005, Seddighin [11] extended the Greub-Rheinboldt inequality (1.9) to pairs of normal operators and established for what vectors the Greub-Rheinboldt inequality becomes equality.

Let $V$ be an $n \times r$ matrix such that $V^{*} V=I_{r}$, i.e., $V$ is suborthogonal. Another wellknown matrix version of the Kantorovich inequality asserts that

$$
V^{*} A^{2} V \leq \frac{(m+M)^{2}}{4 m M}\left(V^{*} A V\right)^{2}
$$

for any $A>0, V^{*} V=I$, and $0<m I<A<M I$.

Mond and Pečarić proved the following matrix version inequality (see (7) in [12]):

$$
\left(V^{*} A^{2} V\right)^{1 / 2}-V^{*} A V \leq \frac{(M-m)^{2}}{4(M-m)} I
$$

for $A>0$ and $V^{*} V=I$. For more related properties and applications, see, e.g., [13-15].

In the next section, we propose some refinements about the matrix Kantorovich-type inequalities (1.2), the Greub-Rheinboldt inequality for commutative positive definite Hermitian matrix pairs, and (1.10) for positive definite matrices, yielding sharpened upper bounds compared with original results, together with an improvement to (1.12).

\section{Main results}

In this section, we first introduce some lemmas.

Lemma 2.1 (in [8], Lemma 2.2) Let $A \in M_{n}$ be a positive definite Hermitian matrix. The following inequalities hold:

$$
\lambda_{1}\|x\|^{2} \leq x^{*} A x \leq \lambda_{n}\|x\|^{2}, \quad 0 \leq\left(\lambda_{n}\|x\|^{2}-x^{*} A x\right)\left(x^{*} A x-\lambda_{1}\|x\|^{2}\right) \leq \frac{1}{4}\left(\lambda_{n}-\lambda_{1}\right)^{2}\|x\|^{4},
$$

and

$$
\begin{aligned}
& \frac{1}{\lambda_{n}}\|x\|^{2} \leq x^{*} A^{-1} x \leq \frac{1}{\lambda_{1}}\|x\|^{2}, \\
& 0 \leq\left(\frac{1}{\lambda_{1}}\|x\|^{2}-x^{*} A^{-1} x\right)\left(x^{*} A^{-1} x-\frac{1}{\lambda_{n}}\|x\|^{2}\right) \leq \frac{\left(\lambda_{n}-\lambda_{1}\right)^{2}}{4\left(\lambda_{1} \lambda_{n}\right)^{2}}\|x\|^{4}
\end{aligned}
$$

for any $x \in \mathbb{C}^{n}$.

Let $A, B$ be two invertible commuting Hermite matrices. Denote by $\lambda_{1} \leq \lambda_{2} \leq \cdots \leq$ $\lambda_{n}$ and $\mu_{1} \leq \mu_{2} \leq \cdots \leq \mu_{n}$ the eigenvalues of $A$ and $B$, respectively. Then there exists a unitary matrix $U \in M_{n}$ such that $A=U \Lambda U^{*}, B=U M U^{*}$, where $\Lambda=\operatorname{diag}\left(\lambda_{1}, \ldots, \lambda_{n}\right)$, $M=\operatorname{diag}\left(\hat{\mu}_{1}, \ldots, \hat{\mu}_{n}\right)$. Note that $\hat{\mu}_{1}, \hat{\mu}_{2}, \ldots, \hat{\mu}_{n}$ is a permutation of $\mu_{1}, \mu_{2}, \ldots, \mu_{n}$. Let $\sigma_{k}=\frac{\lambda_{k}}{\hat{\mu}_{k}}$ $(k=1, \ldots, n)$, then it is easy to see that all eigenvalues of $A B^{-1}$ are $\sigma_{1}, \sigma_{2}, \ldots, \sigma_{n}$. Without 
loss of generality, we may assume that $\sigma_{1}=\min _{k}\left\{\frac{\lambda_{k}}{\hat{\mu}_{k}}\right\}, \sigma_{n}=\max _{k}\left\{\frac{\lambda_{k}}{\hat{\mu}_{k}}\right\}$ and $\sigma_{1} \leq \cdots \leq \sigma_{n}$. For convenience, we introduce the notation

$$
D(A B, x)=x^{*} A\left(\sigma_{n} I-A B^{-1}\right)\left(A B^{-1}-\sigma_{1} I\right) B x .
$$

If $\sigma_{1} \sigma_{n}>0$, then we can define

$$
D\left((A B)^{-1}, x\right)=x^{*} A\left(\frac{1}{\sigma_{1}} I-A^{-1} B\right)\left(A^{-1} B-\frac{1}{\sigma_{n}} I\right) B x .
$$

Lemma 2.2 Let $A$ and $B$ be two positive definite commuting matrices with eigenvalues $0<\lambda_{1} \leq \lambda_{2} \leq \cdots \leq \lambda_{n}, 0<\mu_{1} \leq \mu_{2} \leq \cdots \leq \mu_{n}$, respectively. $\sigma_{1} \leq \sigma_{2} \leq \cdots \leq \sigma_{n}, D(A B, x)$ and $D\left((A B)^{-1}, x\right)$ are as before. Then for any $x \in \mathbb{C}^{n}$,

$$
\begin{aligned}
& 0 \leq D(A B, x) \leq \frac{1}{4}\left(\sigma_{n}-\sigma_{1}\right)^{2}\left|x^{*} A B x\right|, \\
& 0 \leq D\left((A B)^{-1}, x\right) \leq \frac{\left(\sigma_{n}-\sigma_{1}\right)^{2}}{4\left(\sigma_{1} \sigma_{n}\right)^{2}}\left|x^{*} A B x\right|
\end{aligned}
$$

for any $x \in \mathbb{C}^{n}$.

Proof From (2.2),

$$
\begin{aligned}
D(A B, x) & =x^{*} A\left(\sigma_{n} I-A B^{-1}\right)\left(A B^{-1}-\sigma_{1} I\right) B x \\
& =x^{*} U \Lambda U^{*}\left(\sigma_{n} I-U \Lambda U^{*} U M^{-1} U^{*}\right)\left(U \Lambda U^{*} U M^{-1} U^{*}-\sigma_{1} I\right) U M U^{*} x \\
& =x^{*} U \Lambda\left(\sigma_{n} I-\Lambda M^{-1}\right)\left(\Lambda M^{-1}-\sigma_{1} I\right) M U^{*} x .
\end{aligned}
$$

Let $z=\left(z_{1}, \ldots, z_{n}\right)^{T}=(\Lambda M)^{1 / 2} U^{*} x$. Thus, $\|z\|^{2}=z^{*} z=x^{*} U(\Lambda M) U^{*} x=x^{*} A B x$. Then

$$
D(A B, x)=z^{*}\left(\sigma_{n} I-\Lambda M^{-1}\right)\left(\Lambda M^{-1}-\sigma_{1} I\right) z=\sum_{i=1}^{n}\left(\sigma_{n}-\sigma_{i}\right)\left(\sigma_{i}-\sigma_{1}\right) z_{i}^{2} \geq 0
$$

On the other hand,

$$
\sum_{i=1}^{n}\left(\sigma_{n}-\sigma_{i}\right)\left(\sigma_{i}-\sigma_{1}\right) z_{i}^{2} \leq \frac{\left(\sigma_{n}-\sigma_{1}\right)^{2}}{4}\|z\|^{2}
$$

Thus,

$$
D(A B, x) \leq \frac{\left(\sigma_{n}-\sigma_{1}\right)^{2}}{4}\|z\|^{2}=\frac{\left(\sigma_{n}-\sigma_{1}\right)^{2}}{4}\left|x^{*} A B x\right|
$$

The proof of $D\left((A B)^{-1}, x\right)$ is similar.

Theorem 2.3 With the assumptions of Lemma 2.2,

$$
0 \leq \frac{x^{*} A^{2} x \cdot x^{*} B^{2} x}{\left(x^{*} A B x\right)^{2}}-1 \leq \frac{\left(\sigma_{n}-\sigma_{1}\right)^{2}}{4 \sigma_{1} \sigma_{n}}-\frac{1}{\left|x^{*} A B x\right|} \sqrt{D(A B, x) \cdot D\left((A B)^{-1}, x\right)} .
$$


Proof Let $z=(\Lambda M)^{1 / 2} U^{*} x, E=\Lambda M^{-1}=\operatorname{diag}\left(\frac{\lambda_{n}}{\hat{\mu}_{n}}, \ldots, \frac{\lambda_{1}}{\hat{\mu}_{1}}\right)=\operatorname{diag}\left(\sigma_{n}, \ldots, \sigma_{1}\right)$. Then

$$
\frac{x^{*} A^{2} x \cdot x^{*} B^{2} x}{\left(x^{*} A B x\right)^{2}}=\frac{z^{*} E z \cdot z^{*} E^{-1} z}{\left(z^{*} z\right)^{2}} .
$$

From (1.2) and (1.6),

$$
\begin{aligned}
0 & \leq \frac{z^{*} E z \cdot z^{*} E^{-1} z}{\left(z^{*} z\right)^{2}}-1 \leq \frac{\left(\sigma_{n}-\sigma_{1}\right)^{2}}{4 \sigma_{1} \sigma_{n}}-\sqrt{C\left(E, \frac{z}{\|z\|}\right) \cdot C\left(E^{-1}, \frac{z}{\|z\|}\right)} \\
& =\frac{\left(\sigma_{n}-\sigma_{1}\right)^{2}}{4 \sigma_{1} \sigma_{n}}-\frac{1}{\|z\|^{2}} \sqrt{C(E, z) \cdot C\left(E^{-1}, z\right)} .
\end{aligned}
$$

From (2.5) and (2.10), we have

$$
z^{*} z=x^{*} A B x, \quad C(E, z)=D(A B, x), \quad C\left(E^{-1}, z\right)=D\left((A B)^{-1}, x\right) .
$$

By substituting (2.12) and (2.10) into (2.11), the inequality becomes

$$
0 \leq \frac{x^{*} A^{2} x \cdot x^{*} B^{2} x}{\left(x^{*} A B x\right)^{2}}-1 \leq \frac{\left(\sigma_{n}-\sigma_{1}\right)^{2}}{4 \sigma_{1} \sigma_{n}}-\frac{1}{\left|x^{*} A B x\right|} \sqrt{D(A B, x) \cdot D\left((A B)^{-1}, x\right)} .
$$

Corollary 2.4 Let $A$ and $B$ be two positive definite commuting matrices with eigenvalues $0<\lambda_{1} \leq \cdots \leq \lambda_{n}, 0<\mu_{1} \leq \cdots \leq \mu_{n}$, respectively. Then

$$
\frac{x^{*} A^{2} x \cdot x^{*} B^{2} x}{\left(x^{*} A B x\right)^{2}} \leq \frac{\left(\lambda_{1} \mu_{1}+\lambda_{n} \mu_{n}\right)^{2}}{4 \lambda_{1} \mu_{1} \lambda_{n} \mu_{n}}-\frac{1}{\left|x^{*} A B x\right|} \sqrt{D(A B, x) \cdot D\left((A B)^{-1}, x\right)}
$$

holds for any nonzero vector $x \in \mathbb{C}^{n}$.

Proof By Theorem 2.3, we have the following:

$$
0 \leq \frac{x^{*} A^{2} x \cdot x^{*} B^{2} x}{\left(x^{*} A B x\right)^{2}} \leq \frac{\left(\sigma_{1}+\sigma_{n}\right)^{2}}{4 \sigma_{1} \sigma_{n}}-\frac{1}{\left|x^{*} A B x\right|} \sqrt{D(A B, x) \cdot D\left((A B)^{-1}, x\right)} .
$$

Let $f(x)=\frac{(1+x)^{2}}{4 x}$. It can be easily deduced that $f(x)$ is monotone increasing on $[1,+\infty)$. Let $\alpha_{1}=\frac{\mu_{1}}{\lambda_{n}}, \alpha_{n}=\frac{\mu_{n}}{\lambda_{1}}$. From the definition of $\sigma_{1}$ and $\sigma_{n}$, we know that $\frac{\alpha_{n}}{\alpha_{1}} \geq \frac{\sigma_{n}}{\sigma_{1}} \geq 1$. Thus,

$$
\frac{\left(\sigma_{1}+\sigma_{n}\right)^{2}}{4 \sigma_{1} \sigma_{n}}=f\left(\frac{\sigma_{n}}{\sigma_{1}}\right) \leq f\left(\frac{\alpha_{n}}{\alpha_{1}}\right)=\frac{\left(\lambda_{1} \mu_{1}+\lambda_{1} \mu_{1}\right)^{2}}{4 \lambda_{1} \mu_{1} \lambda_{1} \mu_{1}} .
$$

That is,

$$
0 \leq \frac{x^{*} A^{2} x \cdot x^{*} B^{2} x}{\left(x^{*} A B x\right)^{2}} \leq \frac{\left(\lambda_{1} \mu_{1}+\lambda_{1} \mu_{1}\right)^{2}}{4 \lambda_{1} \mu_{1} \lambda_{1} \mu_{1}}-\frac{1}{\left|x^{*} A B x\right|} \sqrt{D(A B, x) \cdot D\left((A B)^{-1}, x\right)} .
$$

Remark From Lemma 2.2 and (2.15), we can obtain a sharpened bound for the classical Kantorovich-type inequality, i.e., the Greub-Rheinboldt inequality. 
Besides the discussion on the Greub-Rheinboldt inequality (1.9), we are also interested in another form of Kantorovich-type inequality aforementioned. We turn our attention to the inequalities (1.11) and (1.12) in the remainder of this paper.

Let $A$ be an $n \times n$ positive (semi-) definite Hermitian matrix with (nonzero) eigenvalues contained in the interval $[m, M]$, where $0<m<M$. Let $V$ be $n \times r$ matrices.

As is declared in (1.11), for $A>0, V^{*} V=I$, and $m, M$ mentioned above, the following inequality holds:

$$
V^{*} A^{2} V \leq \frac{(m+M)^{2}}{4 m M}\left(V^{*} A V\right)^{2}
$$

It is not difficult to see that as $V^{*} V=I$, then $V V^{*}=V V^{+} \leq I$, where + indicates the MoorePenrose inverse. Multiplying from the right and from the left by $V^{*} A$ and $A V$ respectively, we have $V^{*} A^{2} V \geq\left(V^{*} A V\right)^{2}$ for $A>0$. From the well-known Löwner-Heinz inequality, we have $\left(V^{*} A^{2} V\right)^{1 / 2} \geq V^{*} A V$ and the following inequality (see in [16]):

$$
\left(V^{*} A^{2} V\right)^{1 / 2} \leq \frac{m+M}{2 \sqrt{m M}} V^{*} A V
$$

For $z \in[m, M], m>0$, the convexity of $\left(z^{-1}+z / m M\right)$ implies that

$$
z^{-1} \leq \frac{m+M}{m M}-\frac{z}{m M}
$$

If $A$ has the representation $A=\Gamma D_{\alpha} \Gamma^{*}$, where $\Gamma$ is unitary and $D_{\alpha}=\operatorname{diag}\left(\alpha_{1}, \ldots, \alpha_{n}\right)$, and if $0<m \leq \alpha_{i} \leq M, i=1, \ldots, n$, then from (2.16) it follows that

$$
D_{\alpha}^{-1} \leq \frac{m+M}{m M} I-\frac{D_{\alpha}}{m M}
$$

After multiplying from the right and from the left by $\Gamma$ and $\Gamma^{*}$, it is not difficult to see that (2.17) yields the following [17]:

$$
A^{-1} \leq \frac{m+M}{m M} I-\frac{A}{m M}
$$

Based on (2.18), we derive several results on the inequality (1.12).

Theorem 2.5 For any $A>0$ and $V^{*} V=I$,

$$
\left(V^{*} A^{2} V\right)^{1 / 2}-V^{*} A V \leq \frac{(M-m)^{2}}{4(M+m)} I-D^{2}(A, V),
$$

where $D(A, V)=\left(\frac{1}{m+M} V^{*} A^{2} V\right)^{1 / 2}-\frac{(M+m)^{1 / 2}}{2} I$.

Proof From (2.18) and $A>0$, we can get

$$
-A \leq-\frac{m M}{(M+m)} I-\frac{1}{(M+m)} A^{2} .
$$


Since $V^{*} V=I$, (2.20) can be turned into

$$
-V^{\prime \prime} A V \leq-\frac{m M}{(M+m)} I-\frac{1}{(M+m)} V^{\prime \prime} A^{2} V .
$$

By adding $\left(V^{*} A^{2} V\right)^{1 / 2} \geq 0$ to both sides of the inequality (2.21), we obtain that

$$
\left(V^{*} A^{2} V\right)^{1 / 2}-V^{*} A V \leq\left(V^{*} A^{2} V\right)^{1 / 2}-\frac{m M}{(M+m)} I-\frac{1}{(M+m)} V^{*} A^{2} V
$$

i.e.,

$$
\begin{aligned}
\left(V^{*} A^{2} V\right)^{1 / 2}-V^{*} A V & \leq \frac{(M-m)^{2}}{4(M+m)} I-\frac{1}{(M+m)} V^{*} A^{2} V+\left(V^{*} A^{2} V\right)^{1 / 2}-\frac{(M+m)}{4} I \\
& =\frac{(M-m)^{2}}{4(M+m)} I-\left[\left(\frac{1}{M+m} V^{*} A^{2} V\right)^{1 / 2}-\frac{(M+m)^{1 / 2}}{2} I\right]^{2} .
\end{aligned}
$$

Thus, we finally have

$$
\left(V^{*} A^{2} V\right)^{1 / 2}-V^{*} A V \leq \frac{(m-M)^{2}}{4(M+m)} I-D^{2}(A, V),
$$

where $D(A, V)=\left(\frac{1}{(m+M)} V^{\prime \prime} A^{2} V\right)^{1 / 2}-\frac{(M+m)^{1 / 2}}{2} I$.

Remark It is obvious that $D^{2}(A, V) \geq 0$. Thus, Theorem 2.5 indeed presents an improvement of the Kantorovich-type inequality (1.12) in [12].

For an application to the Hadamard product, we have the following corollary.

Corollary 2.6 Let $A_{1}$ and $A_{2}$ be $n \times n$ positive definite matrices with eigenvalues of $A_{1} \otimes A_{2}$ contained in the interval $[m, M]$. Then

$$
\left(A_{1}^{2} \circ A_{2}^{2}\right)^{1 / 2}-A_{1} \circ A_{2} \leq \frac{(M-m)^{2}}{4(m+M)} I-D^{2}\left(A_{1} \otimes A_{2}, V\right),
$$

where $V$ is the selection matrix of order $n^{2} \times n$ with the property $V^{*}\left(A_{1} \otimes A_{2}\right) V=A_{1} \circ A_{2}$ $(\otimes$ and $\circ$ indicate the tensor and the Hadamard product, respectively).

\section{Conclusion}

In this paper, we introduce some new bounds for several Kantorovich-type inequalities for commutative positive definite Hermitian matrix pairs. As a particular situation, in Corollary 2.4, when $A$ and $B$ are both positive definite, the result provides a sharpened upper bound for the matrix version of the well-known Greub-Rheinboldt inequality. Moreover, it holds for negative definite Hermite matrices. Also, a refinement of Kantorovich-type inequalities concerning positive definite matrices is presented together with an application to the Hadamard product. 
Authors' contributions

The authors did not provide this information.

\section{Author details}

'LMIB, School of Mathematics and System Science, Beihang University, Beijing, China. ${ }^{2}$ Faculty of Science and Technology, University of Macau, Macau, C. Postal 3001, China.

\section{Acknowledgements}

The authors would like to thank all the reviewers who read this paper carefully and provided valuable suggestions and comments. This work is supported by the National Natural Science Foundation of China (Grant No. 60831001).

Received: 9 January 2012 Accepted: 10 December 2012 Published: 4 January 2013

\section{References}

1. Greub, W, Rheinboldt, W: On a generalization of an inequality of L.V. Kantorovich. Proc. Am. Math. Soc. 10, 407-415 (1959)

2. Horn, RA, Johnson, CR: Matrix Analysis. Cambridge University Press, Cambridge (1985)

3. Seddighin, M: Antieigenvalue techniques in statistics. Linear Algebra Appl. 430(10), 2566-2580 (2009)

4. Wang, S-G: A matrix version of the Wielandt inequality and its applications to statistics. Linear Algebra Appl. 296(1-3), 171-181 (1999)

5. Householder, AS: The Theory of Matrices in Numerical Analysis. Blaisdell, New York (1964)

6. Nocedal, J: Theory of algorithms for unconstrained optimization. Acta Numer. 1, 199-242 (1992)

7. Dragomir, SS: New inequalities of the Kantorovich type for bounded linear operators in Hilbert spaces. Linear Algebra Appl. 428(11-12), 2750-2760 (2008)

8. Liu, Z, Wang, K, Xu, C: A note on Kantorovich inequality for Hermite matrices. J. Inequal. Appl. 2011, Article ID 245767 (2011)

9. Gustafson, K: Interaction antieigenvalues. J. Math. Anal. Appl. 299(1), 174-185 (2004)

10. Fujii, M, Izumino, S, Nakamoto, R, Seo, Y: Operator inequalities related to Cauchy-Schwarz and Holder-McCarthy inequalities. Nihonkai Math. J. 8(2), 117-122 (1997)

11. Seddighin, M: On the joint antieigenvalue of operators on normal subalgebras. J. Math. Anal. Appl. 312(1), 61-71 (2005)

12. Mond, B, Peăarić, JE: A matrix version of the Ky Fan generalization of the Kantorovich inequality. Linear Multilinear Algebra 36(3), 217-221 (1994)

13. Wang, SG, Shao, J: Constrained Kantorovich inequalities and relative efficiency of least squares. J. Multivar. Anal. 42(2), 284-298 (1992)

14. Chen, L, Zeng, XM: Rate of convergence of a new type Kantorovich variant of Bleimann-Butzerhahn operators. J. Inequal. Appl. 2009, Article ID 852897 (2009)

15. Yuan, JT, Gao, ZS: Complete form of Furuta inequality. Proc. Am. Math. Soc. 136(8), 2859-2867 (2008)

16. Liu, S, Neudecker, H: Several matrix Kantorovich-type inequalities. Aequ. Math. 40(1), 89-93 (1990)

17. Marshall, AW, Olkin, I: Matrix versions of the Cauchy and Kantorovich inequalities. J. Math. Anal. Appl. 197(1), 23-26 (1996)

doi:10.1186/1029-242X-2013-7

Cite this article as: Zhao et al.: Notes on Greub-Rheinboldt inequalities. Journal of Inequalities and Applications 2013 2013:7.

\section{Submit your manuscript to a SpringerOpen ${ }^{\ominus}$ journal and benefit from:}

- Convenient online submission

- Rigorous peer review

- Immediate publication on acceptance

- Open access: articles freely available online

- High visibility within the field

- Retaining the copyright to your article 